\title{
The Analysis of the Courses of the Injury Management and Rescues in Outdoor Sports for the Social Sports Special
}

\author{
Mo Shuangyuan
}

School of Physical education, Guangxi University of Science and Technology, Liuzhou, Guangxi, China (517861250@qq.com)

\begin{abstract}
The outdoor sports is an important part of the social sports. The injury management and rescues is the basic ability for the outdoor sports. So the courses of the injury management and rescues start become foundational course for the social sports special. The teaching aid of the courses is first analyzed from the need of social sports special. Then the lessons content and teaching methods are discussed. And several noticeable points for the courses are proposed. It should be helpful for improving the teaching quality of the course of the injury management and rescues in outdoor sports. And it provides the basis to enhance the students' abilities major in social sports special.
\end{abstract}

Keywords - Courses, injury management, rescues, social sports special

\section{社会体育专业中户外运动损伤处置与救援课程的分析}

\author{
莫双瑗 \\ 广西科技大学体育学院，柳州，广西，中国
}

摘 要 户外运动是社会体育的重要组成部分, 而运动损伤处置与救援是户外运动的基本技能, 所以户外运动损伤处置与救援课 程逐渐成为社会体育专业中的基础性课程。首先从对社会体育专业的要求分析了课程的教学目的, 接着讨论了课程的教学内容和教学 方法, 最后提出了几点教学中值得注意的问题。这将有助于户外运动损伤处置与救援课程教学质量的提高, 同时为社会体育专业学生 能力的提高提供基础。

关键词＼cjkstart课程，损伤处置，救援，社会体育专业

\section{1. 引言}

随着国民经济的不断发展以及生活节奏的加快, 体育 锻炼成为人类日常生活中越来越重要的内容。这就使得对 社会体育指导专业人才的培养越来越受到重视, 所以, 近 年来对于社会体育人才培养的研究逐渐成为高等教育研究 的热点[1][2][3][4]。

在社会体育的发展过程中, 户外运动近年来得到了迅 速发展。特别是随着城市化的不断深入使得人们对于户外 运动的需求越来越大, 这进一步加速了户外运动的推广和 发展。值得注意的是, 由于自然条件和个人身体素质差异 等因素的影响, 户外运动损伤的发生几率相对较高, 这使 得运动损伤处置和救护成为户外运动的基本技能。因此, 在高校社会体育专业中, 户外运动损伤处置与救援的课程 逐渐成为培养学生相关基本技能的基础性专业课, 这也是
相关户外运动专业课程开设的基础。由于户外运动大规模 开展的时间较短, 这一类课程的教学研究并不多, 所以为 提高社会体育专业学生专业素质需要进行相关课程的教学 研究。

2. 课程的教学目的

户外运动损伤处置与救援课程是社会体育专业中一门 培养学生基本能力的课程, 因此在分析课程教学目的时, 需要从社会体育专业学生应具备的专业能力出发, 从人才 培养的定位出发。

按现有的人才培养目标定位, 一般认为社会体育专业 所培养学生需要具有社会体育的基本理论、知识与技能, 毕业已有能够在社会体育领域中从事社会体育活动的组织 管理、咨询指导、经营开发以及教学科研等方面工作。那 
么, 对于户外运动, 就要求社会体育专业学生应具备从事 户外于东组织管理、咨询指导以及相关设备活动经营开发 的能力。显然, 这就要求社会体育专业的学生具备基本的 损伤处置与救援的能力。

根据户外运动的要求, 户外运动损伤处置与救援课程 的教学目标主要包括:

1) 要求学生掌握救护知识和救援技巧的基本知识、基 本方法;

2) 通过课程的学习, 使学生能够在户外活动中规避事 故发生及发生后及时正确的展开自救或者互救, 并能参与 组织相关救援机构展开大规模搜救;

3) 提高学生学生在户外活动中的自救互救能力, 具备 其他户外运动专业课程学习的基础。

\section{3. 课程的教学内容}

根据课程的教学目的, 在课程教学内容安排中需要紧 密联系户外运动的实际情况。以户外运动损伤案例分析为 切入点, 以解决实际问题为目标, 把学科理论的学习融入 对各种情况户外运动损伤处置与救援的研究和认识之中。 根据户外运动常见的运动损伤情况, 结合损伤处置与救援 面对的问题, 可以考虑按以下 15 个部分进行教学内容的组 织。

1) 户外运动救援概述：户外运动救援的作用和意义; 户外运动救援的特点; 户外运动损伤的种类。

2）止血和包扎：血液的特点和作用; 止血技术; 包扎 技术。

3）固定和搬运：骨折损伤的特点；骨折固定技术；伤 员搬运技术。

4）心肺复苏术：心肺复苏的六大步骤; 人工呼吸的注 意事项；心肺复苏终止的条件。

5) 雷击与烧㳷伤: 雷击的特点及预防; 雷击伤的急救; 烧梁伤的急救。

6) 蛇伤急救: 毒蛇的分类与特点; 毒蛇咬伤的判断与 急救; 毒蛇咬伤的预防。

7) 心理救援: 心理救援的作用; 野外心理辅导要点; 野外救援注意事项。

8）绳结技术：绳结技术的作用; 学习八大绳结技术。

9）地形图基础: 地形图的构成要素;标定地图的技巧; 利用地形图行进。

10）营地搭建：营地搭建的原则；营地搭建的技术; 野外营地的管理。

11）药物计划与管理: 户外运动备药原则; 药物管理 规划。
12）野外定向穿越：野外辩向方法; 野外定向穿越。

13）高山伤病防治：高原反应的特点与危害; 高山疾 病的预防与处置; 药物准备。

14）野外搜救：搜救队伍的组建; 人工搜救技巧; 穿 越与呼救方法。

15) 救援机构的管理：救援组织的特点; 救援组织机 构设置; 救援组织的管理

以上 15 个教学内容基本涉及了在户外运动中常见的 损伤处置及搜救需要了解的内容。在具体教学内容的安排 中, 还需要结合学生特点以及教学条件合理安排教学案例, 增强案例对教学内容的支撑, 从而保证对学生损伤处置能 力及参与救援能力的培养, 进而保证该课程相关教学目标 的实现。

另外, 在教学内容的安排上特别值得注意的是, 由于 该课程是户外运动系列课程中的一个部分。相关户外运动 损伤处置与救援的内容可能与其他专业课程存在交叉的可 能, 这就使得对于具体学生、具体院校在课程设置时需要 对具体情况进行分析。如对于 “野外定向穿越”这一类教学 内容, 有的院校将其作为专业选修课开设, 这时在本课程 的教学内容中要适当调整交叉重复的内容。而对于“止血和 包扎”这样的课程, 在本课程的教学中就要突出“户外运动” 的特色, 强调在户外运动的条件下如何处理相关运动损伤。

\section{4. 课程的教学方法}

户外运动损伤处置与救援是一门极为重视实践的课 程。这主要体现在两方面: 一是课程的教学目标是以培养 学生相关能力为主要目的; 二是该课程的教学是相关户外 运动专业课的基础保障。如果不具备相关能力, 无论在后 续户外运动课程的学习实践中, 还是在学生日后组织、参 与户外运动的社会体育活动都存在巨大的安全隐患。这也 是近年来我国相关事故频发的重要原因之一。所以, 针对 这一问题在教学方法上应注重结合实际, 为培养实际能力 进行教学。在具体教学方法上可以考虑以下几点:

\section{1 突出教学目标和教学内容}

每次教学中, 特别是课堂教学中, 一定要突出教学目 标和教学内容。使得学生从上课开始就明确要学习的内容, 通过学习应该掌握的能力。

比如, 在“固定与搬运”的教学中, 首要突出的是“骨 折”, 在上课开始就要让学生明确主要学习如何应对户外运 动出现“骨折”这一损伤。在具体教学过程中可以考虑, 通 过大标题、图片、视频等具有视觉冲击效果的内容刺激学 
生视觉感官, 然后结合教师的讲解明确教学目标和教学内 容。

\section{2 突出对于动作规范化得训练}

在许多损伤处置和救援的过程中有规范化的处置方 式, 为培养学生相关损伤处置的能力在课堂教学中一般会 通过视频、教师示范等方式向学生讲解相关处置动作。在 教学过程中, 特别值得注意学生学习相关动作的规范化问 题。

如在“心肺复苏术”的学习中, 对于胸外按压的手法按 规定是“采用双手叠扣法, 腕肘关节伸直, 利用身体重力, 垂直向下用力按压”。其中对于 “腕肘关节伸直”, 特别是手 肘关节的伸直一直是容易出现错误的地方。因此, 在教学 过程中需要强调这一动作的规范化。在教学过程中, 可以 通过教师示范、学生练习、讲评学生练习、分小组练习、 学生互相检查等多种教学方法进行教学。

特别注意的是, 在教师讲解中一定要强调规范性动作 与不规范性动作的差别, 有条件的可以让学生体会相关差 异。在 “心肺复苏术”胸外按压中, 按规定动作相对省力, 可持续周期长, 针对这一现象可以让学生大量练习, 比较 规范动作与不规范动作的差异。

\section{3 强调学生对实际问题的思考和分析}

根据课程特点, 在实际教学中案例分析是非常重要的 教学方式, 这就使得学生需要对相应情况下的问题做出相 应的判断。在实际教学中, 为培养学生能力一定要强调学 生对实际问题的思考和分析, 在面对实际损伤时能够结合 实际进行思考和分析, 从而为进行合理恰当的处置提供坚 实的基础。

如在“心理救援”的教学中, 可以通过小组讨论、小组 间辩论等多种方式进行案例的分析, 从而增强学生对“心理 救援”中可能出现问题的思考, 进而提高学生 “心理救援”的 能力。

\section{4 在课堂教学中增加有效提问}

在课程的教学中, 为增加学生对课堂讲授内容的思考, 课堂提问是不可缺少的环节。但是提问的过程中, 需要注 意增加有效提问。所谓有效提问是指按教学目标对具体对 象提出问题后能够达到教学要求的提问。在户外运动中主 要是三类提问。

1) 在引入相关损伤、案例分析中引发学生思考的提问。 该类提问应该对提问题干多做解释, 不以有明确答案为目
标, 突出“为什么”的特点, 关键在于引发思考。

2) 在对具体案例进行分析, 在对具体损伤处置动作进 行评价等环节中, 提问以短小精悍为主, 题干清晰明确, 多以判断、选择的方式让学生给出相应答案, 从而了解学 生对相关问题的理解和判断。

3) 在对相关案例进行讲解、对课程内容进行总结时, 提问以回顾为主, 题干要短小精悍, 但是回答内容要求明 确细致, 主要体现为填空、简单的形式。

增加有效提问可以在不同教学环节增强学生对于教学 内容的思考, 从而提高学生应对户外运动损伤的能力。

\section{5. 课程教学中值得注意的问题}

在户外运动损伤处置与救援课程的教学实践中, 针对 课堂教学质量的改善, 以下几个问题是值得注意的。

\section{1 教学辅助手段的使用}

为提高教学效果, 在课程教学中可以引入不少相关教 学辅助手段。如通过动画演示 “蛇伤急救”中蛇毒进入人体 的过程, 在“心肺复苏术”的学习中引入假人进行动作练习。

但是, 值得注意的教学辅助手段仅仅是辅助的, 而教 师在教学过程中的引导作用是不可替代的, 特别是引导学 生思考相关问题的过程中教师有不可替代的作用。

\section{2 教师能力的培养}

教师是户外运动课程安全开展的重要保证。因为在带 领学生去户外运动的过程当中, 如果带队教师不具备救生 的相关能力将是对自己和学生的安全不负责的表现; 如果 开设了户外运动相关课程, 而又不教授户外运动损伤处置 与救援的知识也是极为不安全的。

所以, 该课程是户外运动课程的基础, 而对于师资的 要求较高, 教师应该具有较强的实践经验。但是, 由于户 外运动在中国大规模兴起时间短, 这就使得师资短缺较为 突出。

\section{3 与实践教学的结合}

该课程的开展是以实践为目标的, 对于学生能力的培 养和提高实践教学是最为重要的环节。只有在真实环境下 才能检验和提高学生相关的处置和救援能力, 所以实践教 学是重要的部分。

由于户外运动的损伤处置受户外环境和实际情况影响 较大, 这就使得与实践教学相结合成为本课程不可回避的 问题。 


\section{6. 小结}

针对损伤处置与救援是户外运动基本能力的情况, 对 社会体育专业中开设相关课程进行了分析, 讨论了课程的 教学目的、教学内容和教学方法, 这将有助于相关课程教 学效果的提高, 进而为增强学生相关能力提供基础。

\section{参考文献(References)}

[1] Zhang Liakai. Model construction and composition on sports professional talented persons in 21th century. Journal of Xi'an institute of physical education, 2005, 22(2) pp.108-111.
[2] Wen Zhihong, Wang Shuhong and Chen Li. The cultivating mode of talents for social sports major in Shanxi University. Journal of Jilin institute of physical education. 2012, 28(2), pp. 56-59.

[3] Wang Gangjun, Zhang Yehong, Wang Bochao and Shi Baohong. The reform study and the feasibility analysis about the social sports professional practice teaching pattern. The science education article collects. 2013, (19), pp.150-151.

[4] Ge Yanrong, Jiang Fang. Research on undergraduate curriculum for the major of social sports guidance and management. Journal of environmental management college of China. 2014,(1), pp.69-73 